\title{
Attenuation of specular and diffracted 2D multiples in image space
}

\author{
Gabriel Alvarez ${ }^{1}$, Biondo Biondi ${ }^{1}$, and Antoine Guitton ${ }^{2}$
}

\begin{abstract}
In complex areas, the attenuation of specular and diffracted multiples in image space is an attractive alternative to surface-related multiple elimination (SRME) and to data space Radon filtering. We present the equations that map, via waveequation migration, 2D diffracted and specular water-bottom multiples from data space to image space. We show the equations for both subsurface-offset-domain common-imagegathers (SODCIGs) and angle-domain common-image-gathers (ADCIGs). We demonstrate that when migrated with sediment velocities, the over-migrated multiples map to predictable regions in both SODCIGs and ADCIGs. Specular multiples focus similarly to primaries, whereas diffracted multiples do not. In particular, the apex of the residual moveout curve of diffracted multiples in ADCIGs is not located at the zero aperture angle. We use our equation of the residual moveout of the multiples in ADCIGs to design an apex-shifted Radon transform that maps the 2D ADCIGs into a 3D model space cube whose dimensions are depth, curvature, and apex-shift distance. Well-corrected primaries map to or near the zero-curvature plane and specularly reflected multiples map to or near the zero apex-shift plane. Diffracted multiples map elsewhere in the cube according to their curvature and apex-shift distance. Thus, specularly reflected as well as diffracted multiples can be attenuated simultaneously. We show the application of our apex-shifted Radon transform to a 2D seismic line from the Gulf of Mexico. Diffracted multiples originate at the edges of the salt body and we show that we can successfully attenuate them, along with the specular multiples, in the image Radon domain.
\end{abstract}

\section{INTRODUCTION}

Surface-related multiple elimination (SRME) uses the recorded seismic data to predict and iteratively subtract the multiple series
(Verschuur et al., 1992). Two-dimensional SRME can deal with all kinds of surface-related 2D multiples, provided all relevant data are recorded within the aperture and offset limitations of the survey line. Diffracted multiples from scatterers with a crossline component cannot be predicted by 2D SRME, but in principle they can be predicted by $3 \mathrm{D}$ SRME, provided the acquisition is dense enough and the apertures large enough in both inline and crossline directions. With standard marine streamer acquisition, the sampling in the crossline direction is too coarse and the crossline aperture too small. Diffracted multiples, and multiples from a reflector with crossline dip, need to be removed by other methods (Hargreaves et al., 2003) or the data need to be interpolated and extrapolated to a dense, large aperture grid (van Dedem and Verschuur, 1998; Nekut, 1998).

Radon demultiple in data space (Hampson, 1986; Foster and Mosher, 1992) has proven successful in attenuating specular multiples if the subsurface is not very complex so that primaries are flat after NMO correction in common midpoint (CMP) gathers and the undercorrected multiples can be approximated by parabolas or hyperbolas. In complex subsurface areas, such as under salt, the hyperbolic NMO approximation breaks down. The NMO velocities are inaccurate and therefore, after NMO, the primaries are unlikely to be flat and the residual moveout of the multiples is unlikely to be well represented by parabolas or hyperbolas. The quality of the separation between primaries and multiples in the Radon domain, therefore, deteriorates.

An alternative to SRME and data space Radon demultiple is the attenuation of multiples in the image space. Prestack wave-equation depth migration accurately handles the complex wave propagation of primaries (Sava and Guitton, 2003), provided that the correct migration velocity could have been estimated in the presence of multiples. The residual moveout of primaries in angle-domain commonimage gathers (ADCIGs), therefore, is likely to be flat. It is not immediately obvious, however, what the residual moveout of the overmigrated multiples is in ADCIGs. To maximize the separation of primaries and multiples in the Radon domain, the kernel of the Radon transform should approximate the functional dependency of the residual moveout of the multiples as a function of the aperture angle as

Manuscript received by the Editor December 1, 2006; revised manuscript received April 2, 2007; published online August 14, 2007.

${ }^{1}$ Stanford University, Stanford Exploration Project, Department of Geophysics, Stanford, California. E-mail: gabriel@ sep.stanford.edu biondo@sep. stanford.edu.

23D Geo, Santa Clara, California. E-mail: aguitton@ gmail.com.

(C) 2007 Society of Exploration Geophysicists. All rights reserved. 
much as possible. Sava and Guitton (2003) and Alvarez et al. (2004) used the tangent-squared approximation of Biondi and Symes (2004), assuming that the residual moveout of the multiples is the same as that of primaries migrated with faster velocity. The tangentsquared approximation, however, is a straight ray approximation that is appropriate for primaries that are likely to be only moderately overmigrated. Multiples, on the other hand, given their large difference in velocity with respect to that of the primaries, are likely to be severely overmigrated and the straight ray approximation may not be appropriate for them.

In this paper, we present the equations that describe the mapping of specular and diffracted 2D water-bottom multiples from data space to image space by wave-equation migration in both subsurface-offset-domain common-image-gathers (SODCIGs) and ADCIGs. Primaries are migrated to zero subsurface offsets in SODCIGs and with flat moveout in ADCIGs. We show that specular water-bottom multiples are focused similarly to primaries. Hence, if migrated with constant water velocity, they too are mapped to zero subsurface-offset in SODCIGs and with flat moveout in ADCIGs (Biondi, 2006). In the usual case of migration with velocities faster than water velocity, however, specular water-bottom multiples are overmigrated and thus not mapped to zero subsurface offsets. Instead, they are mapped to subsurface offsets with the opposite sign to that of their surface offsets. We derive the moveout curve of these multiples in SODCIGs and ADCIGs. We then take the special case of the residual moveout of a specular multiple from flat water-bottom in ADCIGs and use it to design a Radon transform that accounts for raybending of the multiple raypath at the multiple-generating interface. This Radon transform improves the separation of primaries and multiples in the Radon domain compared with a Radon transform based on the tangent-squared approximation.

Water-bottom diffracted multiples (even from a flat water-bottom), do not migrate as primary reflections (Alvarez, 2005). That is, they do not focus to zero subsurface offset even if migrated with constant water velocity. These multiples migrate to both positive and negative subsurface offsets in SODCIGs depending on the relative position of the diffractor with respect to the receiver (for receiverside diffracted multiples). In ADCIGs, these multiples have their apex at nonzero aperture angle, similar to their behavior in data space (CMP gathers; Alvarez, 2005). We propose to attenuate these multiples with an apex-shifted Radon transform similar to that used by Alvarez et al. (2004) but replacing the tangent-squared Radon kernel with our new equation for the residual moveout of the multiples in ADCIGS. Apex-shifted transforms have been used for 3D surface multiple prediction (van Dedem and Verschuur, 2002), data

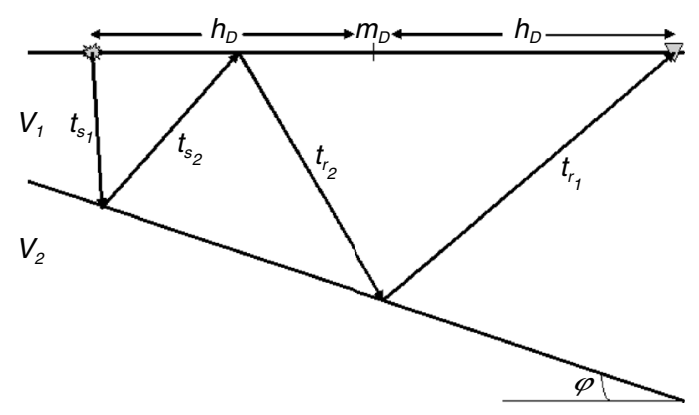

Figure 1. Water-bottom multiple. The subscript $s$ refers to the source and the subscript $r$ to the receiver. interpolation (Trad, 2002), and attenuation of diffracted multiples in data space (Hargreaves et al., 2003).

We expect the real impact of our method for attenuating diffracted multiples to be in $3 \mathrm{D}$ rather than in $2 \mathrm{D}$, though the results that we show in this paper are limited to 2D. Biondi and Tisserant (2004) have presented a method for computing 3D ADCIGs from full 3D prestack migration. These 3D ADCIGs are functions of both the aperture angle and the reflection azimuth. Simple ray tracing modeling shows that out-of-plane multiples map into events with shifted apexes (like the 2D diffracted multiples) and different reflection azimuth than the primaries. We speculate that the attenuation of these multiples from 3D ADCIGs can be accomplished with a methodology similar to the one we present in this paper.

The next section presents the general formalism for the mapping of first-order specular and diffracted water-bottom multiples. We give closed-form equations for the residual moveout of the multiples in SODCIGs and ADCIGs in the special case of a flat water-bottom specular multiple. Parametric equations for diffracted and specular multiples from dipping water-bottom can be found in Alvarez (2005). The following section discusses the design of an apex-shifted Radon transform that uses as kernel our equation for the residual moveout of the specular multiple. The apex-shifted transform is then applied to a simple synthetic data set as well as a real 2D section from the Gulf of Mexico. We then discuss some important practical issues of our approach, and in the last section give our conclusions.

\section{KINEMATICS OF 2D MULTIPLES IN IMAGE SPACE}

In this section, we give the equations that map first-order waterbottom multiple reflections from data space (CMP gathers) to image space (SODCIGs and ADCIGs). We study in detail the special case of a specular multiple from a flat water-bottom. The equation that we derive for the residual moveout of the multiples in ADCIGs for this special case will be the basis for the attenuation of the multiples in the Radon domain. Alvarez (2005) gives parametric equations for other simple cases: specular multiple from a dipping water-bottom and diffracted multiples from flat and dipping water-bottom.

\section{General formulation}

The propagation path of a first-order water-bottom multiple, generated by a planar dipping reflector, as shown in Figure 1, consists of four segments, such that the total traveltime for the multiple is given by

$$
t_{m}=t_{s_{1}}+t_{s_{2}}+t_{r_{2}}+t_{r_{1}}
$$

where the subscript $s$ refers to the source-side rays and the subscript $r$ refers to the receiver-side rays. The data space coordinates are $\left(m_{D}, h_{D}, t_{m}\right)$ where $m_{D}$ is the horizontal position of the common-midpoint (CMP) gather and $h_{D}$ is the half-offset between the source and the receiver.

Wave-equation migration maps the CMP gathers to SODCIGs with coordinates $\left(m_{\xi}, h_{\xi}, z_{\xi}\right)$ where $m_{\xi}$ is the horizontal position of the image gather, and $h_{\xi}$ and $z_{\xi}$ are the half subsurface-offset and the depth of the image, respectively.

As illustrated in Figure 2, at any given depth, the spatial coordinates of the source and receiver rays are given by

$$
x_{s_{\xi}}=m_{D}-h_{D}+V_{1}\left(t_{s_{1}} \sin \alpha_{s}+\rho \tilde{t}_{s_{2}} \sin \beta_{s}\right),
$$




$$
x_{r_{\xi}}=m_{D}+h_{D}-V_{1}\left(t_{r_{1}} \sin \alpha_{r}+\rho \tilde{t}_{r_{2}} \sin \beta_{r}\right),
$$

where $V_{1}$ is the water velocity, $\rho=V_{2} / V_{1}$ with $V_{2}$ the sediment velocity, $\alpha_{s}, \alpha_{r}$ are the takeoff angles of the source and receiver rays with respect to the vertical, $\beta_{s}$ and $\beta_{r}$ are the angles of the refracted source and receiver rays, respectively, and $\tilde{t}_{s_{2}}$ and $\tilde{t}_{r_{2}}$ are the traveltimes of the refracted ray segments. The coordinates of the migrated multiple in the image space are given by:

$$
\begin{gathered}
h_{\xi}=\frac{x_{r_{\xi}}-x_{s_{\xi}}}{2}=h_{D}-\frac{V_{1}}{2}\left[t_{s_{1}} \sin \alpha_{s}+t_{r_{1}} \sin \alpha_{r}\right. \\
\left.+\rho\left(\tilde{t}_{s_{2}} \sin \beta_{s}+\widetilde{t}_{r_{2}} \sin \beta_{r}\right)\right], \\
z_{\xi}=V_{1}\left(t_{s_{1}} \cos \alpha_{s}+\rho \tilde{t}_{s_{2}} \cos \beta_{s}\right) \\
=V_{1}\left(t_{r_{1}} \cos \alpha_{r}+\rho \tilde{t}_{r_{2}} \cos \beta_{r}\right), \\
m_{\xi}=\frac{x_{r_{\xi}}+x_{s_{\xi}}}{2}=m_{D}+\frac{V_{1}}{2}\left(t_{s_{1}} \sin \alpha_{s}-t_{r_{1}} \sin \alpha_{r}\right. \\
\left.+\rho\left(\tilde{t}_{s_{2}} \sin \beta_{s}-\tilde{t}_{r_{2}} \sin \beta_{r}\right)\right),
\end{gathered}
$$

We can compute $\widetilde{t}_{s_{2}}$ and $\widetilde{t}_{r_{2}}$ from the two imaging conditions: (1) at the image point, the depth of both rays has to be the same (because we are computing horizontal subsurface offset gathers) and (2) $t_{s_{2}}+t_{r_{2}}$ $=\tilde{t}_{s_{2}}+\tilde{t}_{r_{2}}$, which follows immediately from equation 1 since at the image point, the total extrapolated time equals the traveltime of the multiple. As shown in Appendix A, the traveltimes of the refracted rays are given by

$$
\begin{aligned}
& \tilde{t}_{s_{2}}=\frac{t_{r_{1}} \cos \alpha_{r}-t_{s_{1}} \cos \alpha_{s}+\rho\left(t_{s_{2}}+t_{r_{2}}\right) \cos \beta_{r}}{\rho\left(\cos \beta_{s}+\cos \beta_{r}\right)}, \\
& \tilde{t}_{r_{2}}=\frac{t_{s_{1}} \cos \alpha_{s}-t_{r_{1}} \cos \alpha_{r}+\rho\left(t_{s_{2}}+t_{r_{2}}\right) \cos \beta_{s}}{\rho\left(\cos \beta_{s}+\cos \beta_{r}\right)} .
\end{aligned}
$$

The refracted angles are related to the takeoff angles by Snell's law: $\sin \left(\beta_{s}+\varphi\right)=\rho \sin \left(\alpha_{s}+\varphi\right)$ and $\sin \left(\beta_{r}-\varphi\right)=\rho \sin \left(\alpha_{r}-\varphi\right)$, from which we get

$$
\sin \beta_{s}=\rho \sin \left(\alpha_{s}+\varphi\right) \cos \varphi-\sqrt{1-\rho^{2} \sin ^{2}\left(\alpha_{s}+\varphi\right)} \sin \varphi,
$$

$\sin \beta_{r}=\rho \sin \left(\alpha_{r}-\varphi\right) \cos \varphi+\sqrt{1-\rho^{2} \sin ^{2}\left(\alpha_{r}-\varphi\right)} \sin \varphi$,

$\cos \beta_{s}=\sqrt{1-\rho^{2} \sin ^{2}\left(\alpha_{s}+\varphi\right)} \cos \varphi+\rho \sin \left(\alpha_{s}+\varphi\right) \sin \varphi$,

$\cos \beta_{r}=\sqrt{1-\rho^{2} \sin ^{2}\left(\alpha_{r}-\varphi\right)} \cos \varphi-\rho \sin \left(\alpha_{r}-\varphi\right) \sin \varphi$.

In ADCIGs, the mapping of the multiples can be directly related to the previous equations by the geometry shown in Figure 2. The halfaperture angle is given by

$$
\gamma=\frac{\beta_{r}+\beta_{s}}{2}
$$

which is the same equation derived for converted waves by Rosales and Biondi (2005). The depth of the image point in ADCIGs $\left(z_{\xi_{\gamma}}\right)$ is given by (Appendix B)

$$
z_{\xi_{\gamma}}=z_{\xi}-h_{\xi} \tan \gamma
$$

Equations 4-6 describe the transformation performed by waveequation migration between CMP gathers $\left(m_{D}, h_{D}, t\right)$ and SODCIGs $\left(m_{\xi}, h_{\xi}, z_{\xi}\right)$. Equations $7-12$ relate the traveltimes and angles of the refracted segments to parameters that can in principle be computed from the data (traveltimes, takeoff angles, reflector dips, and velocities). Equations 13 and 14 provide the transformation from SODCIGs to ADCIGs. These equations are valid for any first-order water-bottom multiple, whether from a flat or dipping water-bottom. They even describe the migration of source-or receiver-side diffracted multiples with the diffractor at the water-bottom, since no assumption has been made relating $\alpha_{r}$ and $\alpha_{s}$ or the individual traveltime segments. They are, however, of little practical use unless we can relate the individual traveltime segments $\left(t_{s_{1}}, t_{s_{2}}, t_{r_{2}}, t_{r_{1}}\right)$, and the angles $\alpha_{s}$ and $\alpha_{r}$ to the known data space coordinates $\left(m_{D}, h_{D}, t_{m}\right)$ and the model parameters $\left(V_{1}, \varphi\right.$, and $\left.\rho\right)$. This may not be easy or even possible analytically for all situations, but it is for the simple but important case of a specular multiple from a flat water-bottom.

\section{Specular multiple from flat water-bottom}

The traveltime of the first-order water-bottom multiple is given by

$$
t_{m}=\frac{4}{V_{1}} \sqrt{\left(\frac{h_{D}}{2}\right)^{2}+Z_{w b}^{2}}=\sqrt{t_{m}^{2}(0)+\left(\frac{2 h_{D}}{V_{1}}\right)^{2}},
$$

where $t_{m}(0)$ is the traveltime of the multiple at zero offset. Notice that $t_{m}$ is the same as the traveltime of a primary at twice the depth of the water-bottom $Z_{w b}=V_{1} t_{m}(0) / 4$.

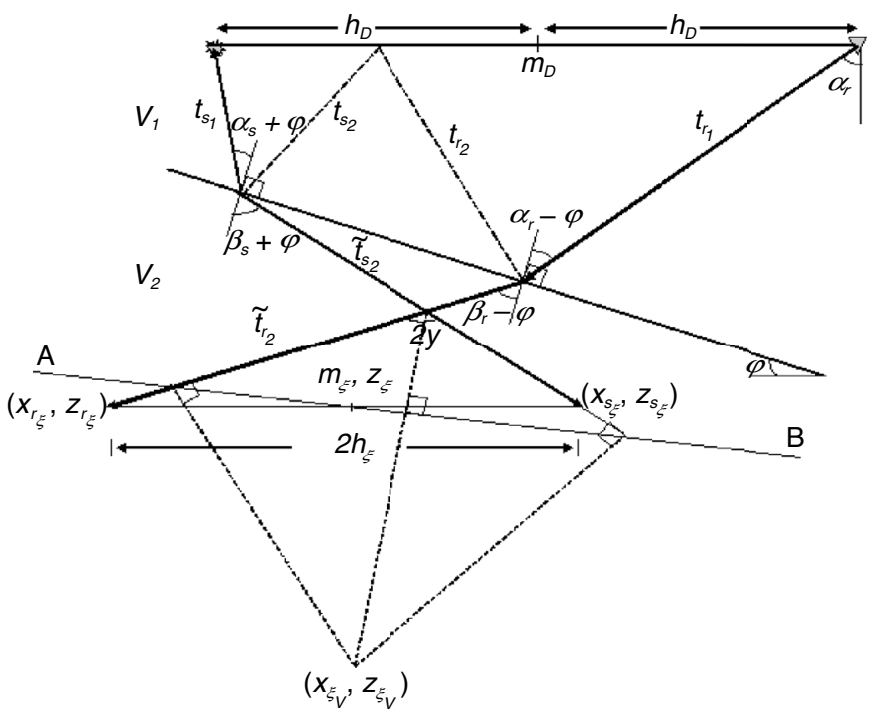

Figure 2. Imaging of water-bottom multiple in SODCIG and ADCIG. The subscript $\xi$ refers to the image point. The line AB represents the apparent reflector at the image point. 
From the symmetry of the problem, $t_{s_{1}}=t_{s_{2}}=t_{r_{1}}=t_{r_{2}}=t_{m} / 4$ and $\alpha_{s}=\alpha_{r}$, which in turn means $\beta_{s}=\beta_{r}$. Furthermore, from equations 7 and 8 , it immediately follows that $\widetilde{t}_{s_{2}}=t_{s_{2}}$ and $\widetilde{t}_{r_{2}}=t_{r_{2}}$, which says that the traveltimes of the refracted rays are equal to the traveltimes of the corresponding segments of the multiple. Equation 4 thus simplifies to

$$
h_{\xi}=\frac{h_{D}}{2}\left(1-\rho^{2}\right) \text {, }
$$

which indicates that the subsurface offset at the image point of a trace with half surface offset $h_{D}$ depends only on the velocity contrast between the water and the sediments. In particular, if the trace is migrated with the water velocity, i.e., $\rho=1$, then $h_{\xi}=0$, which proves our claim that the multiple is imaged exactly as a primary. It should also be noted that, since usually sediment velocity is faster than water velocity, then $\rho^{2}>1$ and therefore the multiples are mapped to subsurface offsets with the opposite sign to that of the surface offset $h_{D}$ when migrated with sediment velocity.

From equation 5, the depth of the image point can be computed as

$$
z_{\xi}=Z_{w b}+\frac{\rho}{2} \sqrt{h_{D}^{2}\left(1-\rho^{2}\right)+4 Z_{w b}^{2}}
$$

which for migration with the water velocity reduces to $z_{\xi}=2 Z_{w b}$, showing that the multiple is migrated as a primary at twice the water depth as is intuitively obvious. Finally, from equation 6 , the horizontal position of the image point reduces to

$$
m_{\xi}=m_{D}
$$

This result shows that the multiple is mapped in the image space to the same horizontal position as the corresponding CMP, even if migrated with sediment velocity. This result is a direct consequence of the symmetry of the raypaths of the multiple reflection in this case. For dipping water-bottom or for diffracted multiples, this is not the case (Alvarez, 2005).

Equations 16-18 give the image space coordinates in terms of the data space coordinates. An important issue is the functional relationship between the subsurface offset and the image depth, because it determines the moveout of the multiples in SODCIGs. Replacing $h_{D}=2 h_{\xi} /\left(1-\rho^{2}\right)$ and $Z_{w b}=z_{\xi}(0) /(1+\rho)$ in equation 17 we get

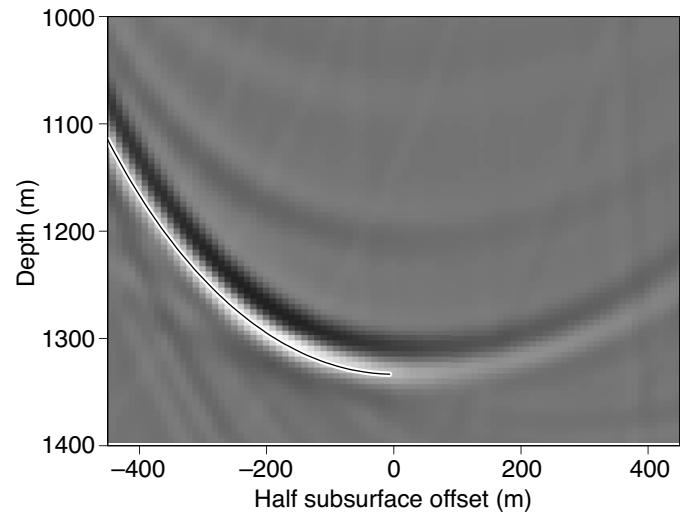

Figure 3. SODCIG of a water-bottom multiple from a flat water-bottom. Water velocity is $1500 \mathrm{~m} / \mathrm{s}$, water depth $500 \mathrm{~m}$, sediment velocity $2500 \mathrm{~m} / \mathrm{s}$, and surface offsets from 0 to $2000 \mathrm{~m}$. Overlaid is the residual moveout curve computed with equation 19.

$$
z_{\xi}=\frac{z_{\xi}(0)}{1+\rho}+\rho \sqrt{\left(\frac{z_{\xi}(0)}{1+\rho}\right)^{2}+\frac{h_{\xi}^{2}}{1-\rho^{2}}} \quad(\rho \neq 1),
$$

which shows that the moveout is a hyperbola (i.e., half of an hyperbola because we already established that $h_{\xi} \leq 0$ if $h_{D} \geq 0$ for off-end geometry).

Figure 3 shows a SODCIG for a specular water-bottom multiple from a flat water-bottom $500-\mathrm{m}$ deep. The data were migrated with a two-layer velocity model: the water layer of $1500 \mathrm{~m} / \mathrm{s}$ and a sediment layer of velocity $2500 \mathrm{~m} / \mathrm{s}$. Larger subsurface offsets (which, according to equation 16, correspond to larger surface offsets) map to shallower depths for the usual situation of $\rho>1$, as we should expect because the rays are refracted to increasingly larger angles until the critical reflection angle is reached. Also notice that the hyperbola is shifted down by a factor $(1+\rho)$ with respect to its image point when migrated with water velocity.

In ADCIGs, the half-aperture angle reduces to $\gamma=\beta_{s}=\beta_{r}$, which in terms of the data space coordinates is given by

$$
\gamma=\sin ^{-1}\left[\frac{2 \rho h_{D}}{V_{1} t_{m}}\right] .
$$

The depth of the image can be computed from equation 14. In particular, if the data are migrated with the velocity of the water, then $\rho$ $=1$, and therefore $z_{\xi \gamma}=2 Z_{w b}$, which means a horizontal line in the $\left(z_{\xi}, \gamma\right)$ plane. Equivalently, we can say that the residual moveout in the $\left(z_{\xi_{\gamma}}, \gamma\right)$ plane is zero, once again corroborating that the water-bottom multiple is migrated as a primary if $\rho=1$. Equation 14 can be expressed in terms of the data space coordinates using equations 16 and 17 and noting that

$$
\tan \gamma=\tan \beta_{s}=\frac{\rho \sin \alpha_{s}}{\sqrt{1-\rho^{2} \sin ^{2} \alpha_{s}}}=\frac{\rho h_{D}}{\sqrt{4 Z_{w b}^{2}+h_{D}^{2}\left(1-\rho^{2}\right)}}
$$

If $\rho=1$, this expression simplifies to $\tan \gamma=h_{D} / 2 Z_{w b}$, which is the aperture angle of a primary at twice the water-bottom depth.

As we did with the SODCIG, it is important to find the functional relationship between $z_{\xi_{\gamma}}$ and $\gamma$ because it dictates the residual moveout of the multiple in the ADCIG. Plugging the equations for the subsurface offset and image depth (equations 16 and 17) into the equation for the depth of the image point in ADCIGs (equation 14), and using the equations for the traveltime of the multiple and the aperture angle (equations 15 and 20) to eliminate $h_{D}$, and simplifying, we get

$$
\begin{aligned}
z_{\xi_{\gamma}} & =Z_{w b}\left[1+\frac{\cos \gamma\left(\rho^{2}-\tan ^{2} \gamma\left(1-\rho^{2}\right)\right)}{\sqrt{\rho^{2}-\sin ^{2} \gamma}}\right] \\
& =\frac{z_{\xi_{\gamma}}(0)}{1+\rho}\left[1+\frac{\cos \gamma\left(\rho^{2}-\tan ^{2} \gamma\left(1-\rho^{2}\right)\right)}{\sqrt{\rho^{2}-\sin ^{2} \gamma}}\right] .
\end{aligned}
$$

Once again, when the multiple is migrated with the water velocity $(\rho=1)$ we get the expected result $z_{\xi_{\gamma}}=z_{\xi_{\gamma}}(0)$, that is, flat moveout (no angular dependence). The residual moveout in ADCIGs is therefore given by 


$$
\begin{aligned}
\Delta n_{\mathrm{RMO}} & =z_{\xi_{\gamma}}(0)-z_{\xi_{\gamma}} \\
& =\left[\rho-\frac{\cos \gamma\left(\rho^{2}-\left(1-\rho^{2}\right) \tan ^{2} \gamma\right)}{\sqrt{\rho^{2}-\sin ^{2} \gamma}}\right] \frac{z_{0}}{1+\rho} .
\end{aligned}
$$

This equation reduces to that of Biondi and Symes (2004) when $\gamma$ is small (Appendix C), which is when we can neglect ray bending at the multiple-generating interface. Figure 4 shows the ADCIG corresponding to the SODCIG shown in Figure 3. Notice that the migrated depth at zero aperture angle is the same as that for the zero subsurface offset in Figure 3. For larger aperture angles, however, the migrated depth increases as indicated in the residual moveout equation 23 . The continuous line corresponds to equation 24 , whereas the dotted line corresponds to the tangent-squared of Biondi and Symes (2004). For large aperture angles, the departure of the straight-ray approximation can be significant.

\section{RADON TRANSFORM}

In this section, we show how to exploit the difference in residual moveout between primaries and multiples in ADCIGs given by equation 24 to design a Radon transform that focuses the primaries and multiples to separate regions of the Radon domain. The general expression for the Radon transform in the angle domain is (Sava and Guitton 2003)

$$
z(q, \gamma)=z_{0}+q g(\gamma)
$$

where $q$ is a measure of curvature and $g(\gamma)$ is the function that approximates the residual moveout of the multiples as a function of the aperture angle $\gamma$. Sava and Guitton (2005) and Alvarez et al. (2004) used the tangent-squared approximation of Biondi and Symes (2004):

$$
g(\gamma)=\tan ^{2} \gamma
$$

but for the focusing of the multiples, a better approximation is derived from equations 24 and 25 :

$$
g(\gamma)=\frac{1}{1+\rho}\left[\frac{\cos \gamma\left(\rho^{2}-\left(1-\rho^{2}\right) \tan ^{2} \gamma\right)}{\sqrt{\rho^{2}-\sin ^{2} \gamma}}-\rho\right] .
$$

This approximation is better because it takes into account ray bending at the multiple-generating interface. This can be seen in Figure 5 ,

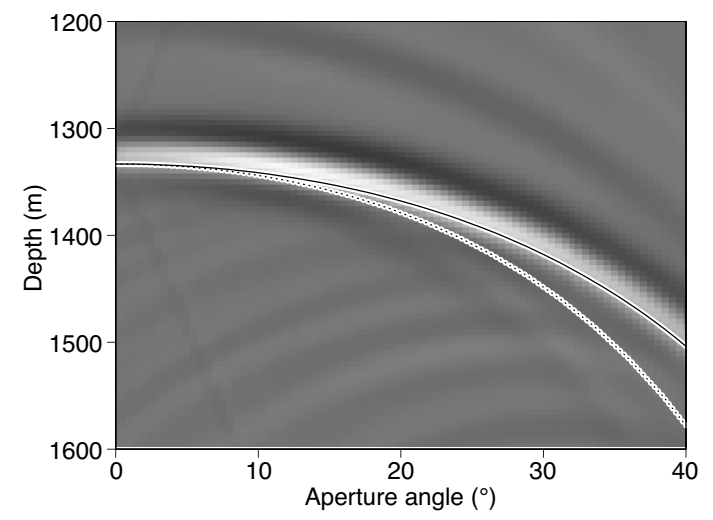

Figure 4. ADCIG for a water-bottom multiple from a two flat-layer model. The dotted curve corresponds to the straight-ray approximation, whereas the solid curve corresponds to the ray-bending approximation. which shows a comparison of the Radon transforms defined be equations 24 (Figure 5b) and A-7 (Figure 5c) applied to a synthetic ADCIG. Notice that the focusing of the primaries does not change because their moveout is zero. The multiples, on the other hand, are better focused with the new transform (Figure 5c), which more closely follows their residual moveout in the ADCIGs. Notice, however, that this synthetic ADCIG has high aperture angles for which the difference between the two approximations is greater. As the an- a)

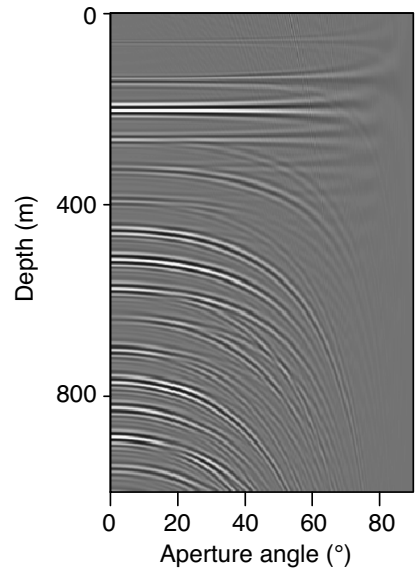

c)

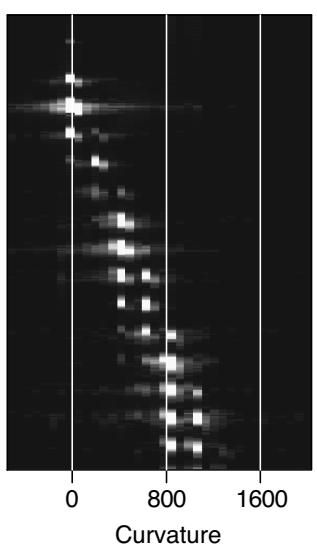

b)

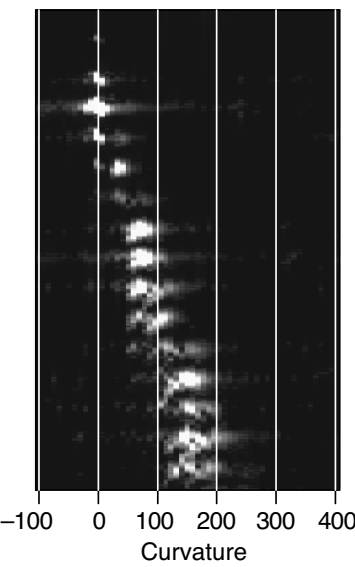

d)

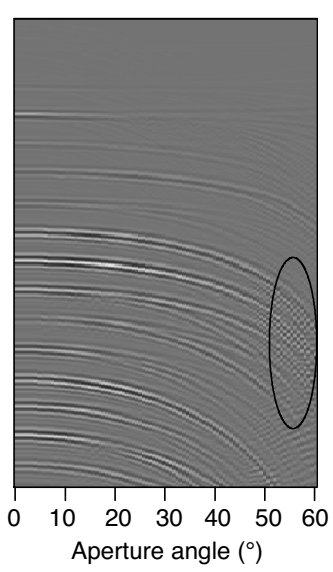

e)

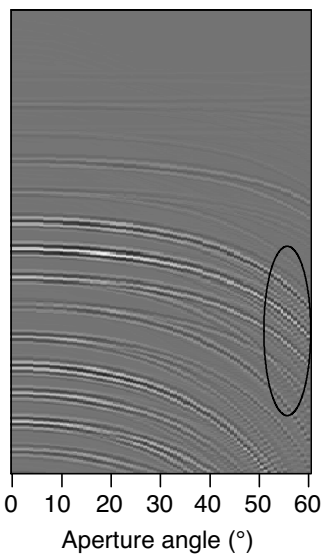

Figure 5. Comparison of Radon transforms for a synthetic ADCIG. (a) The ADCIG; (b) the straight-ray approximation; (c) the raybending approximation. (d) The estimated multiples from (b), and (e) shows the estimated multiples from (c). 
gle coverage decreases, so does this difference. Figure $5 \mathrm{~d}$ and e shows the estimated multiples obtained from the data in Figure $5 \mathrm{~b}$ and c, respectively, after the primaries were muted out. The ovals point to the largest difference, which happens at the larger aperture angles.

\section{Apex-shifted Radon transform}

The apex of the residual moveout curve of the diffracted multiples in ADCIGs is shifted away from zero aperture angle (Alvarez 2005). Therefore, to attenuate the diffracted multiples, we define the transformation from "data" space (ADCIGs) to model space (Radontransformed domain) as

$$
m\left(\Gamma, q, z^{\prime}\right)=\sum_{\gamma} d\left(\gamma, z=z^{\prime}+q g(\gamma-\Gamma)\right),
$$

and from model space to data space as

$$
d(\gamma, z)=\sum_{q} \sum_{\Gamma} m\left(\Gamma, q, z^{\prime}=z-q g(\gamma-\Gamma)\right),
$$

where this time $g(\gamma)$ is given by equation 27 and $\Gamma$ is the lateral apex shift (in units of aperture angle). In this way, we transform the 2D data space of ADCIGs, $d(\gamma, z)$, into a 3D model space, $m\left(\Gamma, q, z^{\prime}\right)$.

In the ideal case of migration with the correct velocity, primaries would be perfectly horizontal in the ADCIGs and would thus map in the model space to the zero-curvature $(q=0)$ plane, i.e., a plane of dimensions depth and apex-shift distance $\left(\Gamma, z^{\prime}\right)$. Specular multiples would map to the zero apex-shift distance $(\Gamma=0)$ plane, i.e., a plane of dimensions depth and curvature $\left(q, z^{\prime}\right)$. Diffracted multiples would map elsewhere in the cube, depending on their curvature and apex-shift distance.

\section{Sparsity constraint}

In order to minimize the number of model space parameters necessary to represent the data in the Radon domain, we implemented the transform given by equations 28 and 29, with the Radon/kernel given by equation 27 , as a least-squares problem with a sparsity constraint. As a linear transformation, the apex-shifted Radon transform can be represented as

$$
\mathbf{d}=\mathbf{L} \mathbf{m},
$$

where $\mathbf{d}$ is the (migrated) data in the angle domain, $\mathbf{m}$ is the model in the Radon domain and $\mathbf{L}$ is the forward apex-shifted Radon trans-

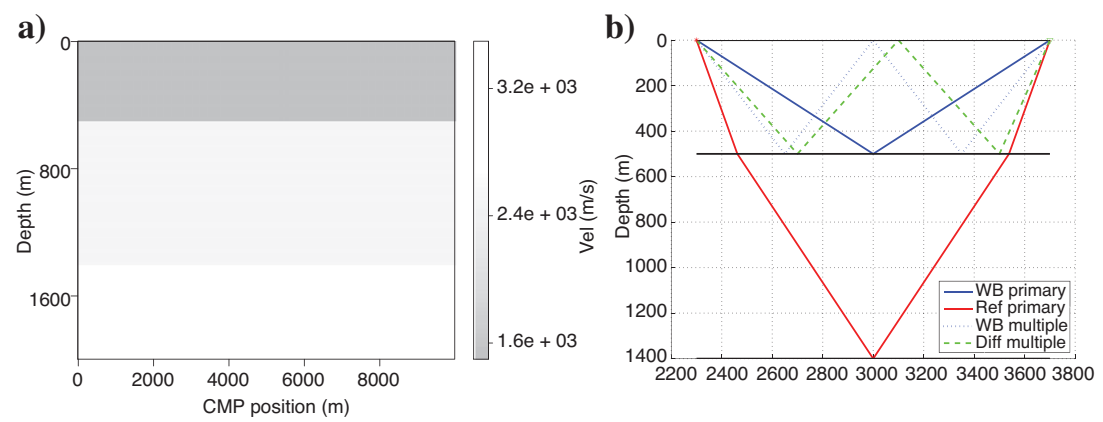

Figure 6. Synthetic model. (a) Velocity model. (b) Raypaths of modeled primaries and multiples. form operator. To find the model $\mathbf{m}$ that best fits the data in a leastsquares sense, we minimize the objective function:

$$
f(\mathbf{m})=\|\mathbf{L} \mathbf{m}-\mathbf{d}\|^{2}+\epsilon^{2} b^{2} \sum_{i=1}^{n} \ln \left(1+\frac{m_{i}^{2}}{b^{2}}\right),
$$

where the second term is a Cauchy regularization that enforces sparseness in the model space (Sacchi and Ulrych, 1996). Here, $n$ is the size of the model space, and $\epsilon$ and $b$ are two constants chosen a priori: $\epsilon$, which controls the amount of sparseness in the model space and $b$, which controls the minimum value below which everything in the Radon domain should be zeroed. The least-squares inverse of $\mathbf{m}$ is given by

$$
\hat{\mathbf{m}}=\left[\mathbf{L}^{\prime} \mathbf{L}+\epsilon^{2} \mathbf{Q}\right]^{-1} \mathbf{L}^{\prime} \mathbf{d},
$$

where $\mathbf{Q}$ is a diagonal matrix whose elements are given by (Sacchi and Ulrych, 1995)

$$
\left(1+\frac{m_{i}^{2}}{b^{2}}\right)^{-1}
$$

Because the model space can be large, we estimate $\mathbf{m}$ iteratively. Notice that the objective function in equation 31 is nonlinear because the model appears in the definition of the regularization term. Therefore, we use a limited-memory quasi-Newton method (Guitton and Symes, 2003) to find the minimum of $f(\mathbf{m})$.

\section{SYNTHETIC DATA EXAMPLE}

In this section, we introduce a simple 2D synthetic example to illustrate the mapping of a water-bottom and a diffracted multiple from data space to image space. Figure 6 shows the velocity model as well as the raypath of the primaries and multiples that were modeled. Figure 7 shows the zero offset section and the CMP gather at CMP position $2400 \mathrm{~m}$. The depth of the deep reflector was chosen so that the multiples and the deep primary came at about the same zerooffset time and were imaged at about the same depth. Notice that the apex of the moveout curve of the diffracted multiple is not at zero offset. Moreover, the moveout curve is not well represented by a hyperbola. Therefore, we cannot attenuate the diffracted multiple via a standard data-space Radon transform.

After prestack wave-equation migration, the primaries are well focused at zero subsurface offset as shown in Figure 8. Figure 8a is the zero-subsurface offset section, whereas Figure $8 \mathrm{~b}$ is the SODCIG taken at CMP location $3800 \mathrm{~m}$. The waterbottom multiple is mapped to the negative subsurface offsets, while the diffracted multiple is mapped to both positive and negative subsurface offsets. Notice that in the zero subsurface offset (Figure 8a), the water-bottom multiple and the deep primary are imaged at about the same depth. After transformation to ADCIGs, the primaries are now flat, whereas the multiples show the expected undercorrected residual moveout (Figure 9a). The apex of the diffracted multiple, however, is not at zero aperture angle. Figure $9 \mathrm{~b}$ shows the Radon plane taken at zero apex shift while Figure $9 \mathrm{c}$ shows the Radon plane taken at the apex-shift of the multiple $\left(14^{\circ}\right)$. Notice that both the prima- 
ries and the water-bottom multiple are well focused in the zero apexshift plane, whereas the diffracted multiple is well focused at its apex-shift plane.

For the sake of comparison, we also applied the non-apex-shifted transform to the data and eliminated the primaries with the same mute pattern. After inverse Radon transforming the multiples and subtracting them from the original ADCIG, we get the results shown in Figure 10. The apex-shifted transform was able to recover the diffracted multiple, although the standard transform mistook it for a specular multiple and thus produced the wrong multiple moveout. Notice that some primary energy leaked into the estimate of the multiples in Figure 10b. Figure 11 shows the estimated primaries. Figure $11 \mathrm{a}$ is the original ADCIG. Figure 11b is the difference between Figure $10 \mathrm{a}$ and $\mathrm{b}$ and therefore is an estimate of the primaries obtained with the apex-shifted transform. Figure 11c is the corresponding estimate with the standard transform. Some residual multiple energy a)

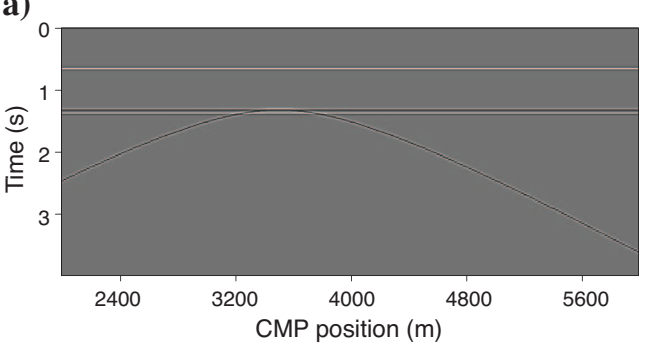

b)

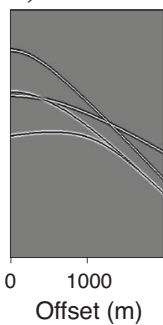

Figure 7. Synthetic data. (a) Zero-offset section. (b) CMP gather at CMP location $2400 \mathrm{~m}$. a)

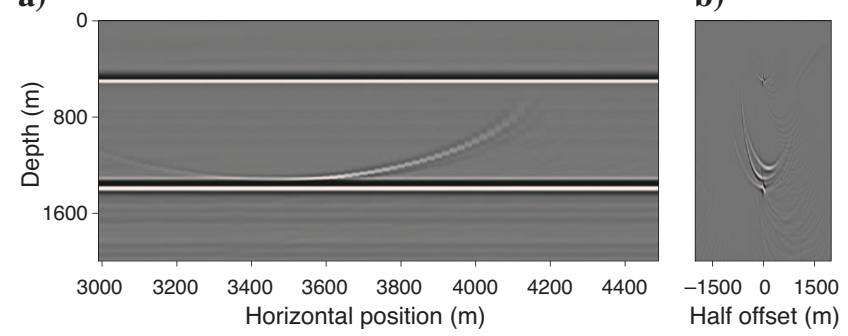

remains above the deep primary in panel (b) but the primary was recovered. The estimation of the primaries could be improved by adaptively matching the estimated multiples to the multiples in the data (as in SRME), before the subtraction. Finally, Figure 11c shows that the poor estimate of the diffracted multiple with the standard transform causes it to leak almost unattenuated into the estimate of the primaries.

\section{GULF OF MEXICO 2D SEISMIC LINE}

In this section, we introduce a seismic line to test our apex-shifted Radon transform with real data. The line is from the Gulf of Mexico and was shot over a large salt body. The presence of the salt creates a host of multiples that obscures any genuine subsalt reflections, as shown in the angle stack of Figure 12. Most multiples are surface-related peg-legs with a leg related to the water-bottom, shallow reflectors, or the top of salt. Below the edges of the salt we also encounter diffracted multiples (indicated by the ovals).

Figure 13 shows four ADCIGs obtained with wave-equation migration as described by Sava and Fomel (2003). Notice that although the data are marine, the ADCIGs show positive and negative aperture angles. We used reciprocity to simulate negative offsets and interpolation to compute the two shortest-offset traces not present in the original data. The CMP gathers were then migrated to angle gathers. The purpose of having both positive and negative aperture angles is to see more clearly the position of the apexes of the diffracted a)

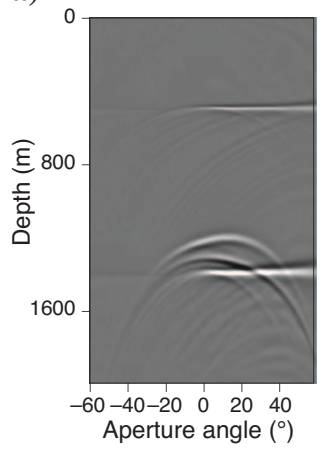

b)

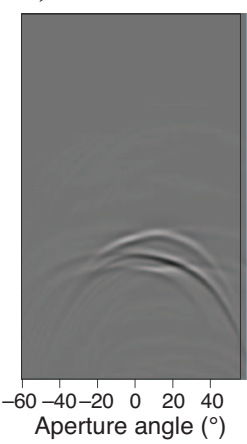

c)

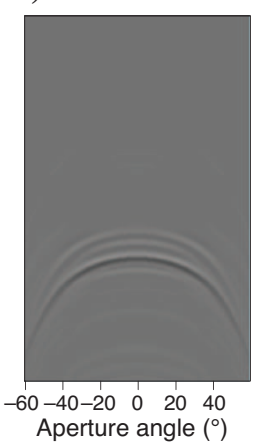

Figure 8. (a) Zero-subsurface offset image and (b) SODCIG at surface location $3800 \mathrm{~m}$. Notice the residual moveout of the diffracted multiple being mapped to both positive and negative offsets. a)

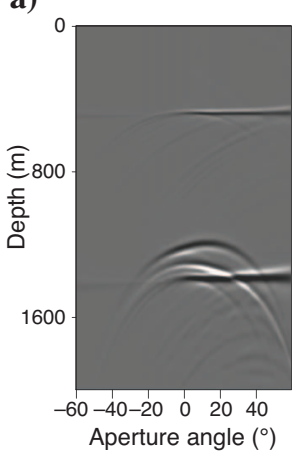

b)

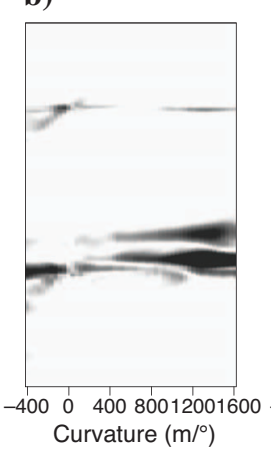

c)

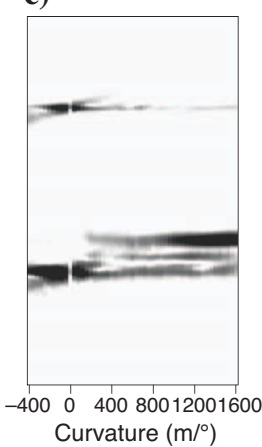

Figure 9. (a) ADCIG of the same SODCIG in Figure 8b; (b) plane taken from the apex-shifted Radon cube at $\Gamma=0$; (c) plane taken from the apex-shifted Radon cube at $\Gamma=10^{\circ}$.
Figure 10. (a) Original ADCIG; (b) estimated multiples with the apex-shifted transform; (c) estimated multiples with the standard transform. a)

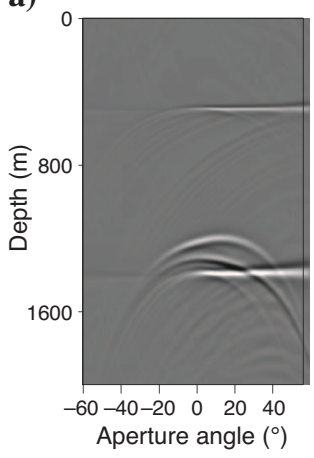

b)

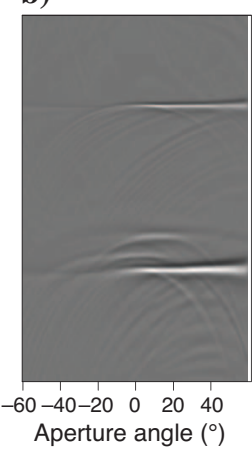

c)

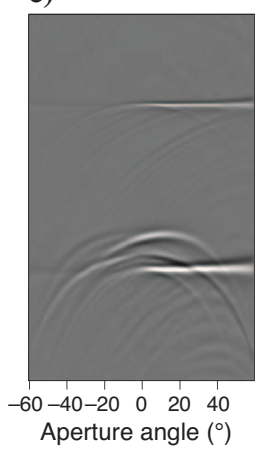

Figure 11. (a) Original ADCIG; (b) estimated primaries with the apex-shifted transform; (c) estimated primaries with the standard transform. 
multiples. The top two ADCIGs correspond to lateral positions directly below the edges of the salt body (CMP positions 6744 and $22056 \mathrm{~m}$ in Figure 12). Notice how the apexes of the diffracted multiples are shifted away from zero aperture angle (e.g., the seagulllooking event at about $4600 \mathrm{~m}$ in Figure 12a). For comparison, Figure $13 \mathrm{a}$ and $\mathrm{b}$ shows two ADCIGs that do not have diffracted multiples. Figure $13 \mathrm{c}$ corresponds to an ADCIG below the sedimentary section (CMP3040 m in Figure 12) and Figure 13d to an ADCIG below the salt body (CMP position $12000 \mathrm{~m}$ in Figure 12). In these ADCIGs, all the multiples are specularly reflected and thus have their apexes at zero aperture angle.

\section{MULTIPLE ATTENUATION RESULTS}

With ideal data, attenuating both specular and diffracted multiples could, in principle, be accomplished by zeroing out (with a suitable

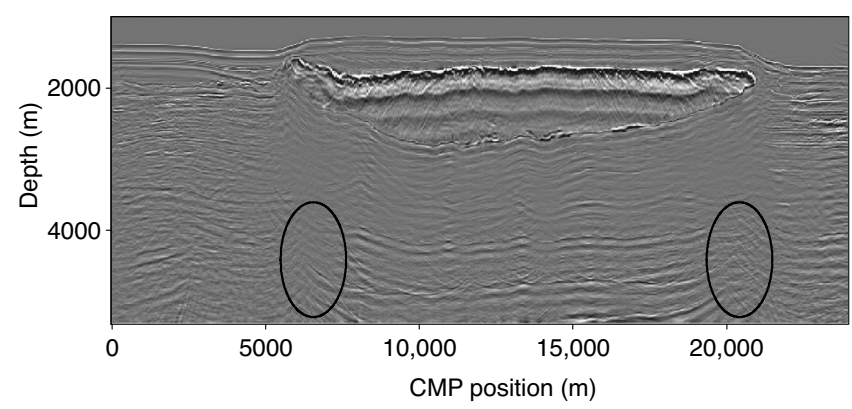

Figure 12. Angle stack of migrated ADCIGs of a 2D seismic line in the Gulf of Mexico. Notice that multiples below the salt obscure any primary reflections.

a)

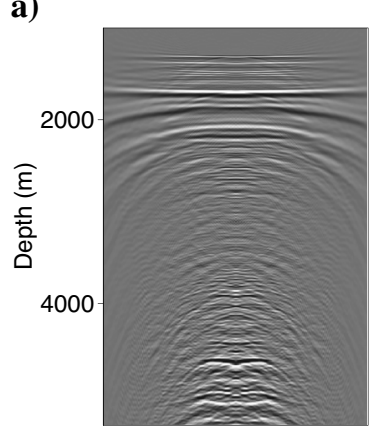

c)

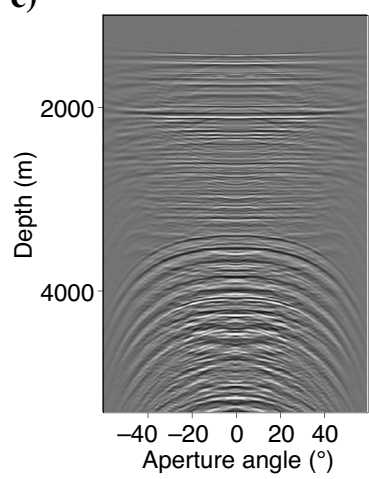

taper) all the $q$-planes except $q=0$ in the model cube $m\left(\Gamma, q, z^{\prime}\right)$ and taking the inverse apex-shifted Radon transform. In practice, however, the primaries may not be correctly migrated and primary energy may map to a few other $q$-planes. Energy from the multiples may also map to those planes and so we have the usual trade-off of primary preservation versus multiple attenuation. The advantage now is that the diffracted multiples are well focused to their corresponding $\Gamma$-planes and can therefore be attenuated.

To illustrate the mapping of the primaries, the specular multiples and the diffracted multiples between the image space $(z, \gamma)$ and the apex-shifted Radon space $\left(\Gamma, q, z^{\prime}\right)$, we chose the ADCIG in Figure 13a. Although this ADCIG shows no discernible primaries below the salt, it nicely shows the apex-shifted moveout of the diffracted multiples. This ADCIG was transformed to the Radon domain with the apex-shifted transform described by equations 28 and 29. The kernel of the Radon transform is given by equation 27 and we applied the Cauchy regularization given in equation 31 . Figure 14 a shows the $\Gamma=0$ plane from the $\left(\Gamma, q, z^{\prime}\right)$ volume. This plane corresponds to zero apex-shift and therefore this is where the majority of the specular multiples should map. Figure $14 \mathrm{~b}$ shows the zero-curvature $q$ $=0$ plane, that is, the plane where the primaries should map. Notice that because the primaries are flat, they are independent of the apexshift $\Gamma$ and therefore map as flat lines on this plane. Notice also that there are no significant primaries on the ADCIG below $2000 \mathrm{~m}$. For comparison, Figure $14 \mathrm{c}$ shows the $\Gamma=8^{\circ}$ plane. This corresponds to the apex-shift of the most obvious diffracted multiple and we see its energy mapped on this plane at about $4000 \mathrm{~m}$. Finally, Figure 14d shows a plane at a large curvature, $q=7200 \mathrm{~m} /$ degree. Notice the energy from the diffracted multiple around $\Gamma=8^{\circ}$. b)

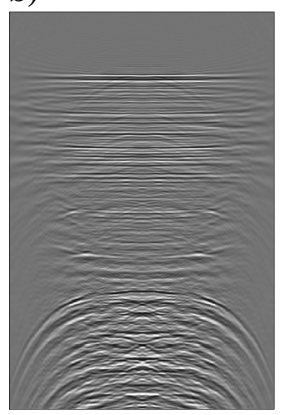

a)

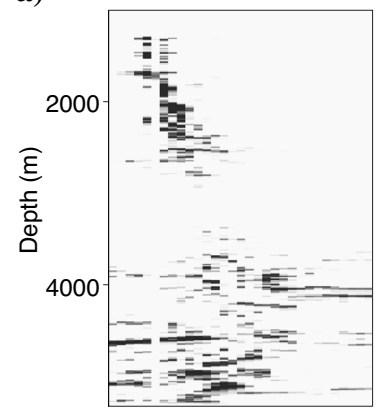

c)

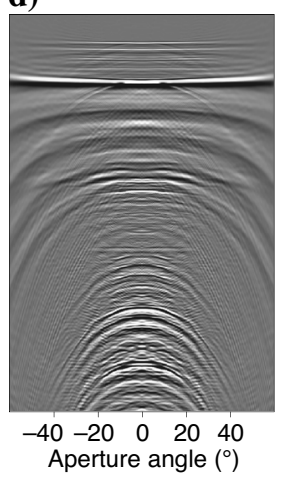

Figure 13. ADCIGs at representative locations along the seismic line. (a) Under the left edge of the salt, CMP at $6744 \mathrm{~m}$; (b) under the right edge of the salt, CMP at 22,056 m; (c) below the sedimentary section, CMP at 3040; (d) below the salt body, CMP at $12000 \mathrm{~m}$.
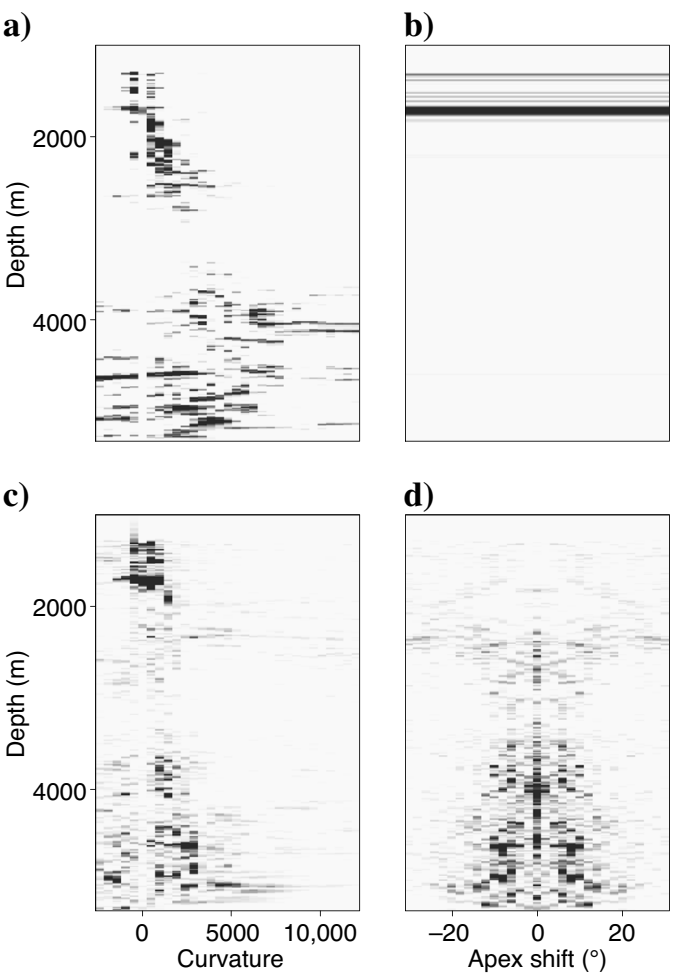

d)

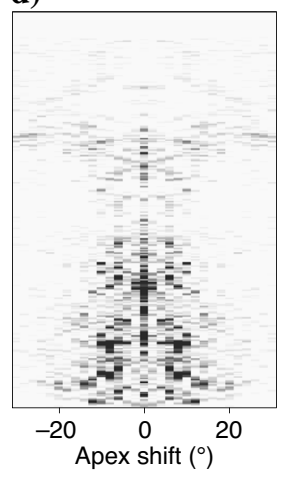

Figure 14. Different views from the cube of the apex-shifted Radon transform for the ADCIG at $6744 \mathrm{~m}$. (a) Zero apex-shift plane; (b) Zero curvature plane; (c) plane at apex shift $\Gamma=8^{\circ}$ and (d) plane at curvature $q=7200 \mathrm{~m} /$ degree. 
It is important to emphasize the difference between the standard transform and the apex-shifted transform. While the $\Gamma=0$ plane of the apex-shifted transform is similar to the standard transform, they are not the same, as shown in Figure 15. Both panels in this figure are plotted with the exact same clip. Primaries are mapped near the $q$ $=0$ line in both planes, while specular multiples are mapped to other $q$ values. Notice how in the standard transform Figure 15a the diffracted-multiple energy is mapped as background noise, especially at the largest positive and negative $q$ values. In the $\Gamma=0$ plane of the apex-shifted transform (Figure 15b), however, the diffracted multiples are not present because their moveout apex is not zero. These multiples, therefore, do not obscure the mapping of the specular multiples. Notice also that the primary energy is much lower than in a)

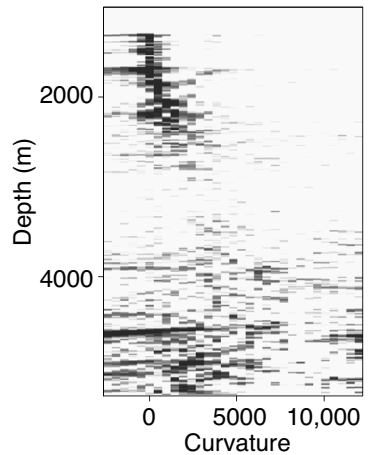

b)

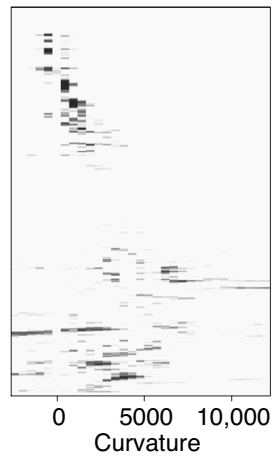

Figure 15. Radon transforms of the ADCIG in Figure 13b. (a) Standard 2D transform and (b) $\Gamma=0$ plane of the apex-shifted 3D transform. (a) and (b) are plotted at the exact same clip value. a)

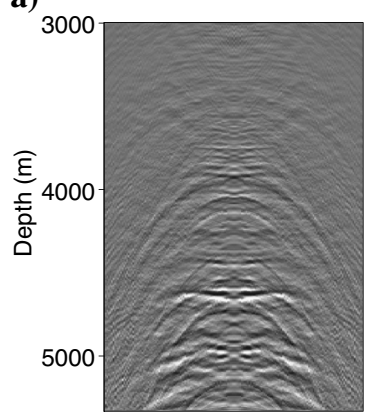

c)

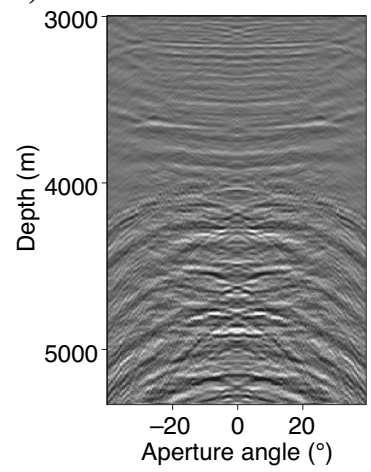

b)

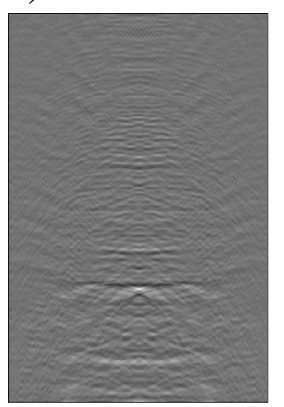

d)

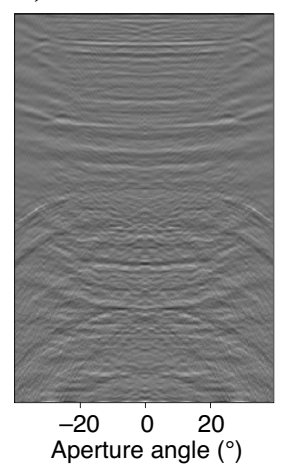

Figure 16. Comparison of primaries extracted with the 2D Radon transform (a) and (c) and with the apex-shifted Radon transform (b) and (d). Notice that some of the diffracted multiples remain in the result with the 2D transform.
Figure 15a because in the apex-shifted transform the primary energy is mapped not only to the $\Gamma=0$ plane but to other $\Gamma$ planes as well, as illustrated previously in Figure 14b.

As with the synthetic data example, rather than suppressing the multiples in the model domain, we chose to suppress the primaries and inverse transform the multiples to ADCIGs. The primaries were then recovered by subtracting the multiples from the data. The multiples were not adaptively matched to the data before subtraction. Figure 16 shows a close-up comparison of the primaries extracted with the standard 2D transform (Sava and Guitton, 2003) and with the apex-shifted Radon transform for the two ADCIGs at the top in Figure 13. The standard transform (Figures 16a and c) was effective in attenuating the specular multiples, but failed at attenuating the diffracted multiples (below $4000 \mathrm{~m}$ ), which are left as residual multiple energy in the primary data. Again, this is a consequence of the apex shift of these multiples. There appears to be no subsalt primary reflections in Figure 16a and $\mathrm{b}$. The flattish reflector at about $4600 \mathrm{~m}$ in Figure $16 \mathrm{~b}$ is actually residual multiple energy (compare with Figure 16a). Similarly, there is only one clearly visible subsalt primary in Figure $16 \mathrm{c}$ and d (about $4200 \mathrm{~m}$ ). This primary was well preserved with both transformations.

Figure 17 shows a similar comparison for the extracted multiples. Notice how the diffracted multiples were correctly identified and extracted by the apex-shifted Radon transform, in Figure $17 \mathrm{~b}$ and d. In contrast, the standard 2D transform misrepresents the diffracted multiples as though they are specular multiples, as seen in Figure 17a and c. We can take advantage of the 3D in the model space of the apex-shifted transform to separate the diffracted multiples from the specular ones. This is shown in Figure 18. a)

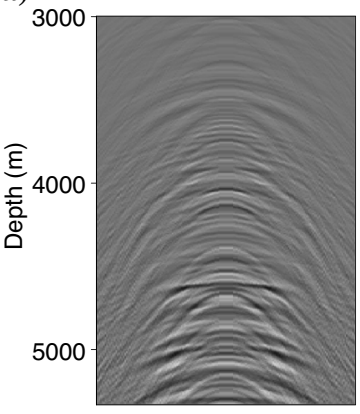

c)

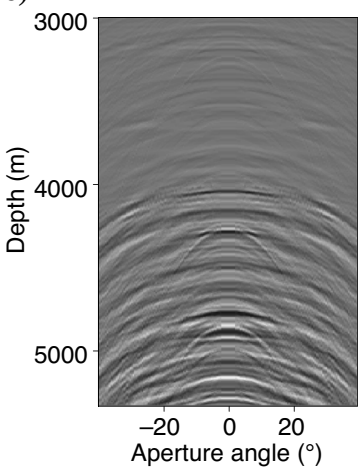

b)

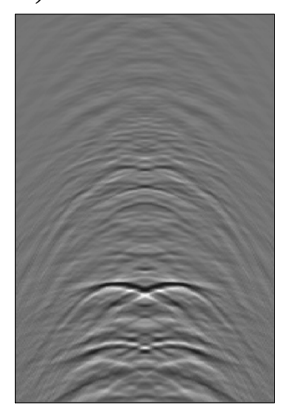

d)

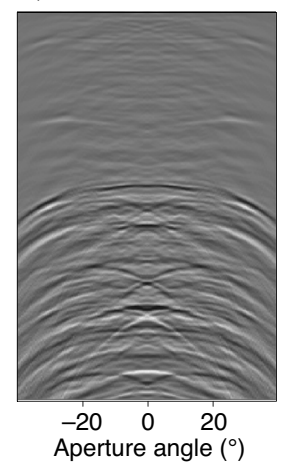

Figure 17. Comparison of multiples extracted with the 2D Radon transform (a) and (c) and with the apex-shifted Radon transform (b) and $(\mathrm{d})$. 
To assess the effect of the improved attenuation of the diffracted multiples on the angle stack, we processed all ADCIGs. Figure 19a shows the angle stack of the data (primaries and multiples) whereas Figure $19 \mathrm{~b}$ and $\mathrm{c}$ shows the primaries extracted with the standard Radon transform and with the apex-shifted Radon transform, respectively. All panels are plotted with the exact same clip value. Both transforms are effective at attenuating the specular multiples, with the apex-shift transform providing improved attenuation of the diffracted multiples (indicated by the ovals). This is further illustrated in Figure 20, it shows the difference between the primaries extracted with both transforms (Figure 19b and c). This figure is blank above $2800 \mathrm{~m}$ because the mute pattern was applied only below this depth (there are no shallower multiples). Figure 19a and b are therefore exactly the same down to 2800-m depth.

It is somewhat surprising that the large differences between the prestack estimates of the multiples obtained with the standard transform and the apex-shifted transform translate to relatively minor differences in the angle stacks of the estimated primaries. The reason is the well-known power of the stack itself to attenuate events with high curvature relative to flat events. Because the main difference between the estimated primaries obtained with both transforms is in the high curvature events (recall Figures 16 and 17), after the stack the improvement in attenuation of the diffracted multiples with the apex-shifted transform is not nearly as large as in the ADCIGs. Having cleaner ADCIGs, however, is important for velocity analysis, illumination studies, and other applications. Using the apex-shifted transform is particularly important in those cases.

With this data set, it is very difficult to identify any primary reflections below the edge of the salt, so it is hard to assess if the primaries have been equally preserved with both transforms. It is known, however, that for this data set, there are no multiples above a depth of about $3600 \mathrm{~m}$, between CMP positions 0 to $5000 \mathrm{~m}$. The fact that the difference panel (Figure 20) appears nearly blank in that zone shows that introducing the extra apex-shift dimension to improve the attenuation of the diffracted multiples did not affect the extraction of the primaries compared with the standard transform. It should be emphasized, however, that any method of multiple attenuation, and in particular those based on moveout differences with the primaries, has the risk of attenuating some primaries along with the multiples. In this case, of course, this is especially true for those primaries that were not accurately imaged, so that their moveout in the ADCIGs was not entirely flat. Weak subsalt primaries, in particular, may a)

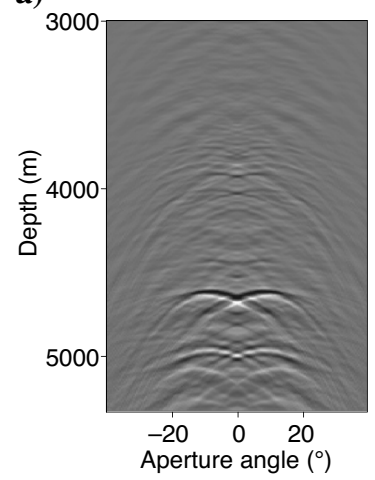

b)

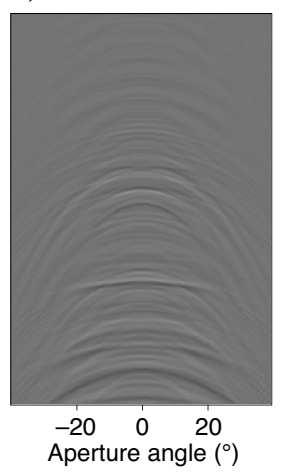

Figure 18. Comparison of (a) diffracted and (b) specular multiples for the ADCIG in Figure 13a. Notice the lateral shifts in the apexes of the diffracted multiples. not have been well imaged because of inaccuracies in the migration velocity field and $3 \mathrm{D}$ effects. These primaries, therefore, may have been attenuated with both the standard and the apex-shifted Radon transforms.

Let us now compare the angle stacks of the estimated multiples obtained with both transforms. Figure 21 is similar to Figure 19 for the multiples. The main difference is in the diffracted multiples, as highlighted by the ovals.
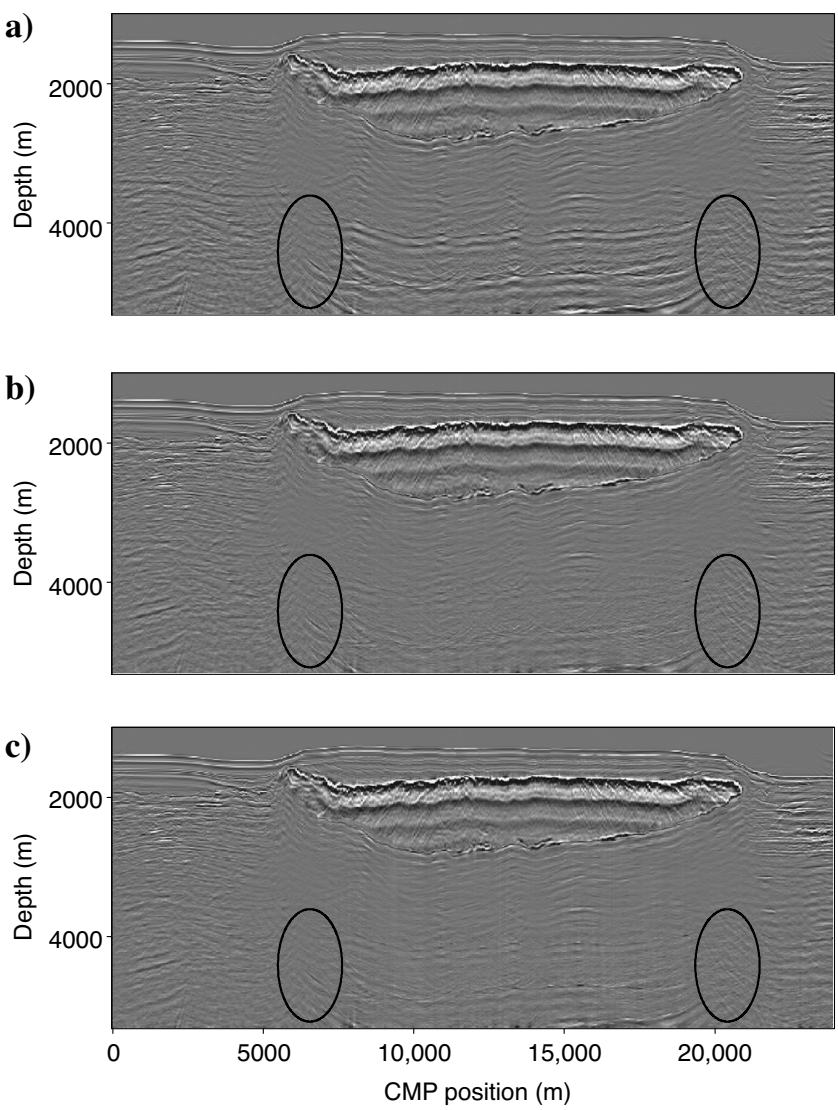

Figure 19. Comparison of angle stacks for primaries. (a) The angle stack of the multiples and primaries. (b) The angle stack of the primaries obtained with the standard transform, whereas (c) is the angle stack of the primaries obtained with the apex-shifted transform.

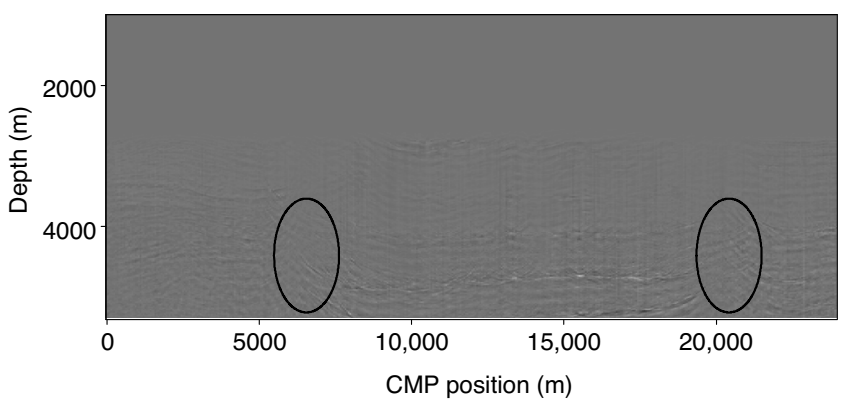

Figure 20. Difference between the primaries obtained with the standard transform minus the primaries obtained with the apex-shifted transform. 


\section{DISCUSSION}

In this section, we discuss some important practical aspects of our method. We start, however, with a discussion on the relative merits of attenuating the multiples in image space as opposed to the industry standard of attenuating them in data space.

\section{Image space versus data space}

In principle, attenuating the multiples in data space has the important advantage that the estimation of the migration velocity field is not affected by the presence of the multiples. This is only true, however, provided that the level of attenuation is such that no significant residual multiple energy remains (that could be mistaken as primaries) and that the primaries are unaffected by the attenuation of the multiples. As discussed before, this is unlikely to be the case for data-space Radon filtering when the subsurface is complex. Furthermore, any residual multiple energy will distort the imaging of the primaries resulting in a deficient final image.

SRME can effectively attenuate all multiples with a bounce at the water surface, but only if all necessary data are collected. In virtually all instances of real 3D data acquisition, the crossline sampling is too coarse, the crossline aperture is too small, the short offsets are missing, and acquisition obstacles and cable feathering produce irregular geometry. The data need to be interpolated and extrapolated to satisfy the requirements of 3D SRME. This is not a trivial endeavor and the performance of SRME greatly depends on it. Moreover, diffracted multiples and specular multiples from an interface with steep crossline dip may have bounce points well outside the crossline aperture, making them hard or impossible to predict.

Attenuating the multiples in image space solves the problem of the complex wave propagation of primaries and multiples. Prestack wave-equation migration takes care of the complexity of the wavefield propagation and makes the primaries very likely to be flat in ADCIGs and therefore more easily separable from the multiples in the Radon domain. No data interpolation or extrapolation is necessary because no multiples are predicted. Since very accurate migration velocities are always necessary to get a good depth image, however, postponing the multiple attenuation step until after migration does not come without a price. Computation of an accurate migration velocity field may be compromised by the presence of the multiples.

\section{Sensitivity to errors in migration velocities}

From the point of view of the attenuation of the multiples, it is important that the migration velocities be accurate enough for the primaries to be flat and the multiples to exhibit residual moveout in ADCIGs. This is likely to be the case for water-bottom and peg-leg multiples because water velocity is very different from sediment velocity, but may not be so for internal multiples. Therefore, the method is likely to be successful in attenuating water-bottoms and peg-leg multiples even if there are small errors in the estimation of the migration velocity field. The attenuation of internal multiples will require more accurate migration velocities.

\section{Radon transform parameters}

The performance of the Radon transform to focus the primaries and multiples to separate regions of the transformed domain depends on the choice of curvature parameters and apex-shift values.
In particular, curvatures should range from small negative values to allow for the possibility of slightly undermigrated primaries to large enough values to accommodate the maximum curvatures of the overmigrated multiples. We have found that these are not particularly critical parameters as long as the curvature sampling is fine enough to avoid aliasing in the Radon domain. This is also true for the apex-shift parameters. They are not critical, because their role is only to provide room for the mapping of the diffracted multiples, thus preventing them from interfering with the primaries and the specular multiples that map to the zero apex-shift plane. A critical step is the design of the mute pattern to eliminate the primaries and keep the multiples. There are several ways that this could be implemented. We constructed a mask of ones for the multiple regions and zeros for the primary region, smoothed it laterally and in depth, and multiplied it by the transformed data.

An important and somewhat difficult parameter to estimate is the one that controls the Cauchy regularization (parameter $b$ in equation 33). We want the data in the ADCIG to be explained in the Radon domain by as few parameters as possible, but avoiding the risk of attenuating the contribution from weak subsalt primaries. This is a trial and error parameter and it requires some testing to get a satisfactory value.

\section{Comment on diffracted multiples}

The results shown in the previous section demonstrate that with the apex-shifted Radon transform it is possible to attenuate, although not completely remove, the diffracted multiples. It should be noted,
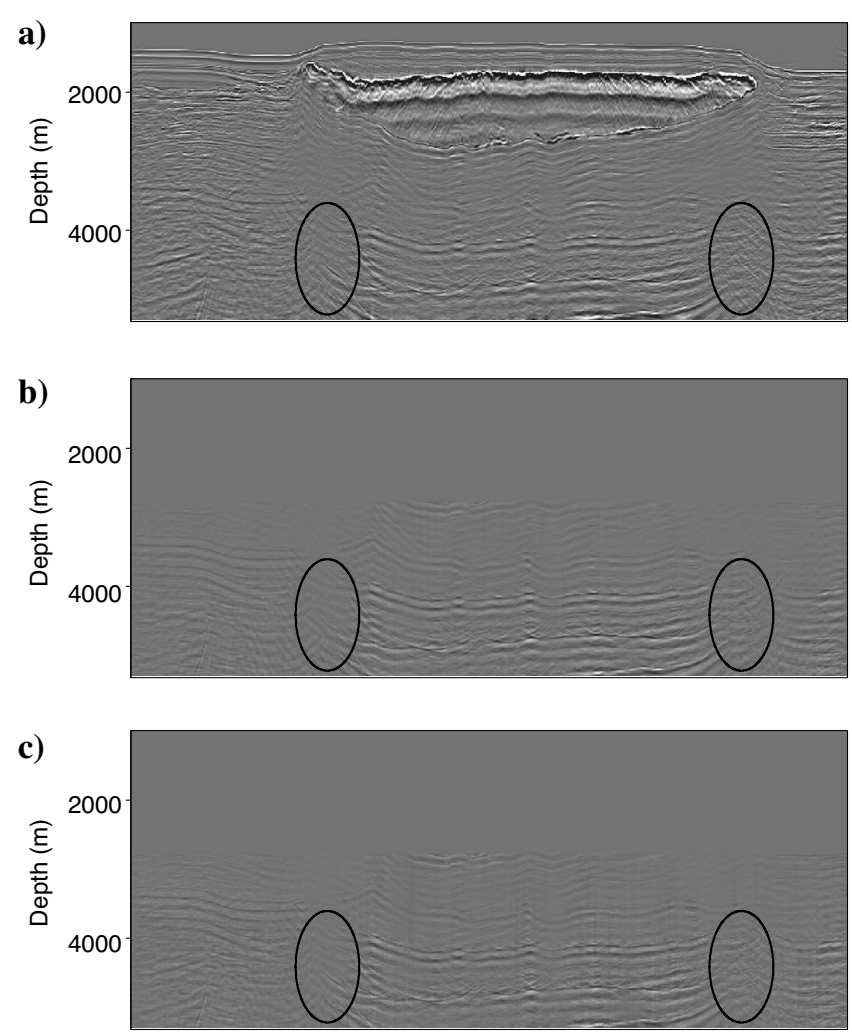

Figure 21. Comparison of angle stacks for multiples. (a) The angle stack of the multiples and primaries. (b) The angle stack of the multiples obtained with the standard transform; (c) the angle stack of the multiples obtained with the apex-shifted transform. 
however, that in our seismic section it is very difficult to find a legitimate primary reflection below the salt and, in particular, below the edge of the salt, where the contamination by the diffracted multiples is stronger. It is somewhat disappointing that the attenuation of the diffracted multiples did not help in uncovering any meaningful primary reflections in our example. We expect the situation to be different with other data sets.

We should also emphasize that adding the extra dimension to deal with the diffracted multiples does not in itself resolve the usual trade-off between primary preservation and multiple attenuation. We saw this limitation in this case, which forced us to let some residual multiple energy leak into the extracted primaries. The flatter the primaries are in the ADCIGs, and the more accurately the kernel of the Radon transform approximates the residual moveout of the multiples, the better are our chances to reduce the residual multiple energy.

\section{Extension to 3D data}

With 3D data, the ADCIGs are a function of the reflection azimuth as well as the aperture angle (Biondi and Tisserant, 2004). The ADCIGs, therefore, are three dimensional and even for the specular multiples the Radon transform will also be three dimensional. For the diffracted multiples, the apex shift is a function of both the aperture angle and the reflection azimuth and the Radon transform would be more complicated. This is a subject of current research.

\section{CONCLUSIONS}

To attenuate multiples in the image space via filtering in the Radon domain, we need an accurate representation of their residual moveouts in either SODCIGs or ADCIGs. Accounting for ray bending at the multiple-generating interface increases the focusing power of the Radon transform and therefore the separation between primaries and multiples.

The apex-shifted Radon transform in ADCIGs to map from $(z, \gamma)$ to $\left(z^{\prime}, q, \Gamma\right)$ is effective in attenuating specular and diffracted multiples in 2D marine data. The residual moveout of both multiples in ADCIGs is well behaved and the extra dimension provided by the apex shift improves the attenuation of the diffracted multiples. This is particularly important in applications such as velocity analysis, where having ADCIGs as free of multiples as possible is very important.

\section{ACKNOWLEDGMENTS}

We thank WesternGeco for providing the data set, the sponsors of the Stanford Exploration Project for their support to carry out this study, and the associate editor and the anonymous reviewers for many helpful suggestions.

\section{APPENDIX A TRAVELTIME OF REFRACTED RAYS}

In this section we derive traveltime equations 7 and 8 . From the equation for the depth of the image point (equation 5) we have:

$$
t_{s_{1}} \cos \alpha_{s}+\rho \widetilde{t}_{s_{2}} \cos \beta_{s}=t_{r_{1}} \cos \alpha_{r}+\rho \widetilde{t}_{r_{2}} \cos \beta_{r},
$$

and from the imaging condition (the sum of the traveltime of the extrapolated rays at the image point has to be equal to the traveltime of the multiple) we have

$$
t_{s_{2}}+t_{r_{2}}=\tilde{t}_{s_{2}}+\tilde{t}_{r_{2}}
$$

Solving those two equations for $\tilde{t}_{s_{2}}$ and $\tilde{t}_{r_{2}}$ we get

$$
\begin{aligned}
& \tilde{t}_{s_{2}}=\frac{t_{r_{1}} \cos \alpha_{r}-t_{s_{1}} \cos \alpha_{s}+\rho\left(t_{s_{2}}+t_{r_{2}}\right) \cos \beta_{r}}{\rho\left(\cos \beta_{s}+\cos \beta_{r}\right)}, \\
& \tilde{t}_{r_{2}}=\frac{t_{s_{1}} \cos \alpha_{s}-t_{r_{1}} \cos \alpha_{r}+\rho\left(t_{s_{2}}+t_{r_{2}}\right) \cos \beta_{s}}{\rho\left(\cos \beta_{s}+\cos \beta_{r}\right)} .
\end{aligned}
$$

It is interesting to check these equations in two particular cases. For a specular multiple from a flat water-bottom, we have $\alpha_{s}=\alpha_{r}$, $\beta_{s}=\beta_{r}, t_{s_{1}}=t_{s_{2}}=t_{r_{2}}=t_{r_{1}}$, and therefore we get $\widetilde{t}_{s_{2}}=t_{s_{2}}$ and $\widetilde{t}_{r_{2}}=t_{r_{2}}$ as the geometry of the problem requires. Notice that this is true for any $\rho$. The second case is for a specular water-bottom multiple migrated with water velocity $(\rho=1)$. In that case, $\beta_{s}=\alpha_{s}$ and $\beta_{r}=\alpha_{r}$. Furthermore, because the multiple behaves as a primary, $\left(t_{s_{1}}+t_{s_{2}}\right) \cos \alpha_{s}=\left(t_{r_{1}}+t_{r_{2}}\right) \cos \alpha_{r}$ and we again get $\tilde{t}_{s_{2}}=t_{s_{2}}$ and $\tilde{t}_{r_{2}}=t_{r_{2}}$.

\section{APPENDIX B}

\section{IMAGE DEPTH IN ADCIGS}

Figure B-1 shows the basic construction to compute the image depth in ADCIGs based on the image depth in SOCIGs. Triangles $\mathrm{ABD}$ and $\mathrm{CBD}$ are congruent, because they have one side common and the other equal because $|\mathrm{AB}|=|\mathrm{BC}|=h_{\xi}$. Therefore, $\theta=\pi / 2$ $-\beta_{r}+\delta$. Also, triangles AED and FCD are congruent because $|\mathrm{AD}|=|\mathrm{CD}|$ and also $|\mathrm{AE}|=|\mathrm{CF}|$ (Biondi and Symes, 2004). Therefore, the angle $\delta$ in triangle DCF is the same as in triangle AED. We can compute $\delta$ from the condition

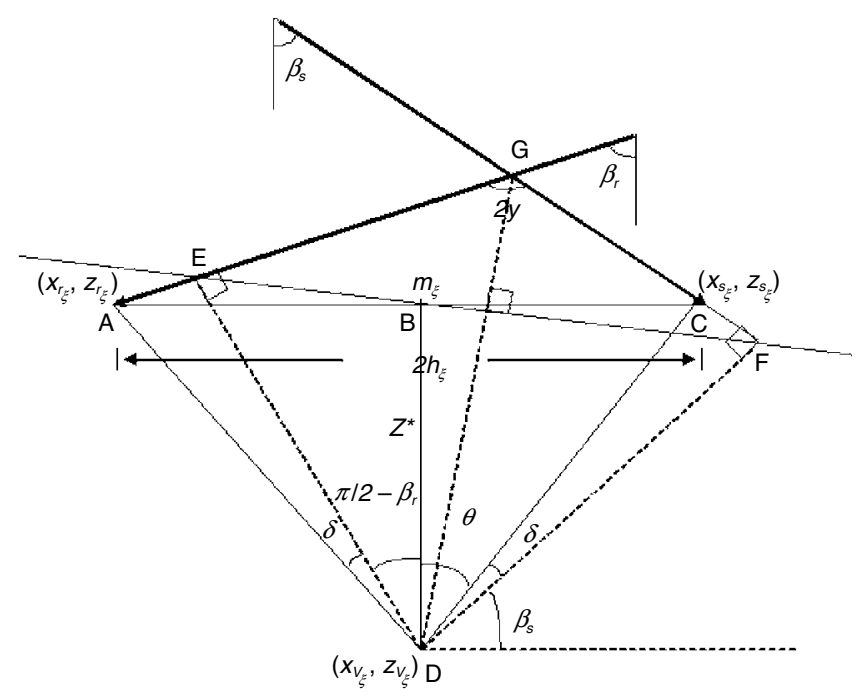

Figure B-1. Sketch to show the computation of the image depth in an ADCIG. 


$$
\begin{aligned}
\theta+\delta+\beta_{s} & =\frac{\pi}{2}, \\
\frac{\pi}{2}-\beta_{r}+\delta+\delta+\beta_{s} & =\frac{\pi}{2}, \\
\delta & =\frac{\beta_{r}-\beta_{s}}{2} .
\end{aligned}
$$

The depth of the image point in the ADCIG, from triangle ABC, is therefore

$$
z_{\xi_{\gamma}}=z_{\xi}+z^{*}=z_{\xi}+\left(\operatorname{sign}\left(h_{\xi}\right)\right) h_{\xi} \cot \left(\frac{\pi}{2}-\beta_{r}+\delta\right) .
$$

Replacing the expression for $\delta$ we get, after some simplification (and taking $\left.\operatorname{sign}\left(h_{\xi}\right)=-1\right)$

$$
z_{\xi_{\gamma}}=z_{\xi}+z^{*}=z_{\xi}-h_{\xi} \tan \left(\frac{\beta_{r}+\beta_{s}}{2}\right)=z_{\xi}-h_{\xi} \tan (\gamma) .
$$

\section{APPENDIX C}

\section{RESIDUAL MOVEOUT IN ADCIGS}

In this appendix we show that, for a flat reflector, the residual moveout of the multiples in ADCIGs reduces to the tangent-squared expression derived by Biondi and Symes (2004) for the residual moveout of undermigrated primaries:

$$
\Delta \mathbf{n}_{\mathrm{RMO}}=(\rho-1) \tan ^{2} \gamma z_{0} \mathbf{n} .
$$

Start with equation 23

$$
z_{\xi_{\gamma}}=\frac{z_{\xi_{\gamma}}(0)}{1+\rho}\left[1+\frac{\cos \gamma\left(\rho^{2}-\left(1-\rho^{2}\right) \tan ^{2} \gamma\right)}{\sqrt{\rho^{2}-\sin ^{2} \gamma}}\right],
$$

where $z_{\xi_{\gamma}}(0)$ is the normal-incidence migrated-depth, (i.e., $\left.z_{0}\right)$ in the previous equation.

There is an important and unfortunate difference in notation here, however, because $\rho$ in equation $\mathrm{C}-1$ is the ratio of the migration to the true slowness whereas $\rho$ in equation $\mathrm{C}-2$ is the ratio of the migration to the true velocity. Therefore, in order to get a better idea of how the approximation for the RMO of the multiples (accounting for ray bending at the reflector interface) relates to that of the primaries (neglecting ray bending), we rewrite equation $\mathrm{C}-2$ replacing $\rho$ by $1 / \rho$ and $z_{\xi_{\gamma}}(0)$ with $z_{0}$ to get:

$$
z_{\xi_{\gamma}}=\left[\rho+\frac{\cos \gamma\left(1-\left(\rho^{2}-1\right) \tan ^{2} \gamma\right)}{\sqrt{1-\rho^{2} \sin ^{2} \gamma}}\right] \frac{z_{0}}{1+\rho} .
$$

Because $\Delta n_{\mathrm{RMO}}=z_{0}-z_{\xi_{\gamma}}$, we get:

$$
\Delta n_{\mathrm{RMO}}=\left[1-\frac{\cos \gamma\left(1-\left(\rho^{2}-1\right) \tan ^{2} \gamma\right)}{\sqrt{1-\rho^{2} \sin ^{2} \gamma}}\right] \frac{z_{0}}{1+\rho} .
$$

For small $\gamma, \sin \gamma \approx 0$ and $\cos \gamma \approx 1$, therefore

$$
\Delta n_{\mathrm{RMO}}=\left(\rho^{2}-1\right) \tan ^{2} \gamma \frac{z_{0}}{1+\rho}=(\rho-1) \tan ^{2} \gamma z_{0} .
$$

This is the same as equation C-1, save for the unit vector $\mathbf{n}$. This result is intuitively appealing because it shows that the approximation of neglecting ray bending at the reflecting interface deteriorates as the aperture angle increases, which is when the ray bending is larger.

\section{REFERENCES}

Alvarez, G., 2005, Mapping of water-bottom and diffracted 2D multiple reflections to image space: Stanford Exploration Project, SEP-123, 129-154.

Alvarez, G., B. Biondi, and A. Guitton, 2004, Attenuation of diffracted multiples in angle-domain common-image gathers: 74th Annual International Meeting, SEG, Expanded Abstracts, 1301-1304.

Biondi, B., 2006, 3D seismic imaging: SEG.

Biondi, B., and W. Symes, 2004, Angle-domain common-image gathers for migration velocity analysis by wavefield-continuation imaging: Geophysics, 69, 1283-1298.

Biondi, B., and T. Tisserant, 2004, 3D angle-domain common-image gathers for migration velocity analysis: Geophysical Prospecting, 62, 575-591.

Foster, D., and C. Mosher, 1992, Suppression of multiple reflections using the radon transform: Geophysics, 57, 386-395.

Guitton, A., and W. Symes, 2003, Robust inversion of seismic data using the huber norm: Geophysics, 68, 1310-1319.

Hampson, D., 1986, Inverse velocity stacking for multiple elimination: Canadian Journal of Exploration Geophysicists, 22, 44-55.

Hargreaves, N., B. VerWest, R. Wombell, and D. Trad, 2003, Multiple attenuation using an apex-shifted Radon transform: 74th Annual International Meeting, SEG, Expanded Abstracts, 1929-1932.

Nekut, A., 1998, 3D surface-related multiple elimination: 68th Annual International Meeting, SEG, Expanded Abstracts, 1511-1514.

Rosales, D., and R. Biondi, 2005, Converted-waves angle-domain commonimage gathers: 75th Annual International Meeting, SEG, Expanded Abstracts, 959-962.

Sacchi, M., and T. Ulrych, 1995, High-resolution velocity gathers and offset space reconstruction: Geophysics, 60, 1169-1177.

, 1996, Estimation of the discrete fourier transform as a linear inversion approach: Geophysics, 61, 4, 1128-1136.

Sava, P., and S. Fomel, 2003, Angle-domain common-image gathers by wavefield continuation methods: Geophysics, 68, 1065-1074.

Sava, P., and A. Guitton, 2003, Multiple attenuation in the image space: 73rd Annual International Meeting, SEG, Expanded Abstracts, 1933-1936. , 2005, Multiple attenuation in the image space: Geophysics, 70, no. 1, V10-V20.

Trad, D., 2002, Interpolation with migration operators: 72nd Annual International Meeting, SEG, Expanded Abstracts, 2174-2177.

van Dedem, E., and D. Verschuur, 1998, 3D surface-related multiple elimination and interpolation: 68th Annual International Meeting, SEG, Expanded Abstracts, 1321-1324.

, 2002, 3D surface-related multiple prediction using sparse inversion: 71st Annual International Meeting, SEG, Expanded Abstracts, 1285 1288

Verschuur, D., A. Berkhout, and C. Wapenaar, 1992, Adaptive surface-related multiple elimination: Geophysics, 57, 1166-1167. 